\section{A transgenic mouse model of dementia with Lewy bodies suggests a link between $\alpha$-synuclein expression, presynaptic vesicle pathology and cognitive deficit}

\section{Walter J Schulz-Schaeffer}

Prion \& Dementia Research Unit, Department of Neuropathology, University Medical Center Göttingen, Robert-Koch-Str.40, 37075 Göttingen, Germany mwjschulz@med.uni-goettingen.de

Evaluation of: Lim Y, Kehm VM, Lee EB et al. $\alpha$-syn suppression reverses synaptic and memory defects in a mouse model of dementia with Lewy bodies. J. Neurosci. 31, 10076-10087 (2011). This study reports on the regional and time-dependent pathological changes of mice expressing human wild-type or A53T-mutant $\alpha$-synuclein under the control of a CaMKII $\alpha$ promoter as well as a tetracyclineregulated transactivator. The transgene was turned on from postnatal day 21 onwards. A53T-mutant $\alpha$-synuclein expression leads to a dementia with Lewy body-like phenotype. A presynaptic $\alpha$-synuclein-related pathology goes handin-hand with memory impairment as measured by conditioned fear. By turning off the $\alpha$-synuclein transgene at a particular time point after a synaptic pathology has already been developed, a regression of the $\alpha$-synuclein-related changes can be observed and the memory impairment does not progress or even mildly improves.

This article reviews a recent publication by Lim and colleagues [1]. Although dementia with Lewy bodies (DLB) and Parkinson's disease (PD) have been neuropathologically diagnosed by the presence and amount of Lewy bodies, which mainly contain aggregated $\alpha$-synuclein [2], the pathophysiological link between $\alpha$-synuclein aggregation and neurodegeneration is not well understood. It seems to be most unlikely that the process of neurodegeneration can be explained by the presence of cellular Lewy bodies alone (reviewed in [3]). In addition to Lewy bodies, $\alpha$-synuclein accumulation is observed in Lewy neurites in neuronal processes, as well as in synapses [4]. The clinical symptoms may be best explained by a synaptic pathology. The decrease of neurotransmitter release is a striking feature in PD and DLB. In this context, it is of special interest that mild overexpression of $\alpha$-synuclein leads to synaptic dysfunction due to defective synaptic vesicle turnover $[5,6]$. Genetic studies clearly indicate that an elevated $\alpha$-synuclein level accelerates the development of PD in a dose-dependent manner. However, the link between $\alpha$-synuclein expression, the observed $\alpha$-synuclein aggregation and neurodegeneration (i.e., dementia) remains to be elucidated.

\section{Methods \& results}

Mice expressing human wild-type or $A 53 T$ mutant $\alpha$-synuclein under the control of a CaMKII $\alpha$ promoter and a tetracycline-regulated transactivator were generated as a 'tet-off' system. This system makes possible the suppression of the transgene expression by feeding the mice with doxycycline. Due to previous experience in which overexpression of human wild-type or $A 53 T$-mutant $\alpha$-synuclein led to degeneration of postmitotic neurons in the hippocampal dentate gyrus during postnatal development [7], expression was suppressed from the observed day of insemination until postnatal day 21.

Lim and coworkers reported abnormal immunohistochemical $\alpha$-synuclein staining of the $A 53 T$-mutant $\alpha$-synuclein-transgenic mice from 4 months of age and older. In the limbic areas, cingulated cortex, hippocampus and mammillary body, the authors observed a dotted granular pattern of $\alpha$-synuclein accumulation without perikaryal inclusions that was more pronounced at 8 months of age. The same was seen in the olfactory bulb and the subthalamic nucleus, but not in neocortical regions. The pattern of $\alpha$-synuclein reactivity was interpreted as a synaptic or neuritic localization. Interestingly,
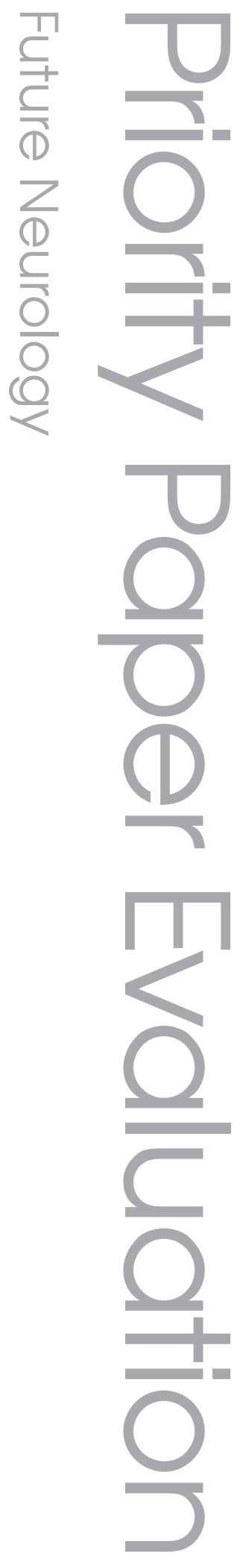

\section{Keywords}

- $\alpha$-synuclein $=$ cognition n dementia with Lewy bodies

Future $\because$ Medicine part of 
a limited $\alpha$-synuclein pathology was observed in the brainstem and spinal cord, suggesting a neuritic spread to the neural projections from the olfactory and limbic systems.

In 20-22-month-old A53T-mutant $\alpha$-synuclein-transgenic mice, a wide distribution of cytoplasmic $\alpha$-synuclein accumulation and marked nerve cell loss was observed. Human wild-type $\alpha$-synuclein-transgenic mice developed limbic and brainstem pathology, although later and less extensively, but not the hippocampal pathology and the severe nerve cell loss seen in $A 53 T$-mutant $\alpha$-synuclein-transgenic mice. Biochemical analyses by serial extraction using buffers of increasing strength and thioflavin $S$ staining indicated that the amount of insoluble $\alpha$-synuclein increases with age and cytoplasmic pathology and that noncytoplasmic $\alpha$-synuclein accumulation does not react with thioflavin S.

An ongoing debate exists regarding whether the abnormally accumulated $\alpha$-synuclein is post translationally modified and whether this is important for the pathophysiology of disease. To distinguish Ser-129-phosphorylated from unphosphorylated $\alpha$-synuclein, a polyclonal antibody was generated that specifically detects the unphosphorylated form (Syn1150). This antibody recognizes only amino acid residues 125 to 129 of $\alpha$-synuclein and was used complementarily to antibody P-syn/81A which detects Ser-129 phosphorylation.

In 12 -month-old $A 53 T$-mutant $\alpha$-synuclein transgenic-mice, only a part of the $\alpha$-synuclein accumulation was detectable by the P-syn/81A antibody. Interestingly, three patterns were recognized: a co-labeling of $\alpha$-synuclein pathology by both antibodies with marked overlap in the cingulated cortex; a co-labeling with nearly no overlap in the hippocampus; and a lack of labeling of phosphorylated $\alpha$-synuclein in the mammillary body, where unphosphorylated $\alpha$-synuclein pathology was present. At 20 months of age, an increase in Ser-129 phosphorylation of $\alpha$-synuclein pathology in all mentioned regions was reported to be clear.

Is hippocampal $A 53 T$-mutant $\alpha$-synuclein accumulation related to cognitive impairment? Lim and coworkers used a contextual fear-conditioning test that requires an intact hippocampal function and a cued fear conditioning test that depends on an intact amygdala, but less on an intact hippocampus [1]. Whereas the $A 53 T$-mutant $\alpha$-synuclein mice at 8 months of age showed a significantly impaired contextual memory compared with different control groups, a nonsignificant trend towards impairment was seen for the cued fear memory. Human wild-type $\alpha$-synuclein-transgenic mice up to 8 months of age showed no changes compared with controls. The behavioral tests correlate well with the morphological changes, as was shown by a negative correlation between a freezing score rating the contextual fear memory and semiquantification of $\alpha$-synuclein pathology in the hippocampus.

The inducible $\alpha$-synuclein-transgenic mouse model allows the authors to address the question of whether the pathological changes can be halted or even reversed by the withdrawal of $\alpha$-synuclein. Human A53T-mutant $\alpha$-synuclein-transgenic mice were given a doxycycline diet for 3 months, starting at 9 months of age, and pathological changes in the hippocampus were compared with 8-and 12-month-old mice without suppression of transgenic $\alpha$-synuclein expression. In the latter, $\alpha$-synuclein pathology was detectable in the oriens layer and stratum radiatum of $\mathrm{CA} 1$, the stratum lucidum of CA3 and the molecular layer of the dentate gyrus, which progressed in severity with age. This $\alpha$-synuclein pathology was not detectable in mice in which the $\alpha$-synuclein transgene was suppressed between 9 and 12 months of age.

Does $\alpha$-synuclein suppression have a detectable effect on synapses? Lim and coworkers examined the expression of synaptic vesicle proteins in CA3 mossy fiber terminals [1]. Synaptophysin, synaptotagmin, CSP- $\alpha$, synapsin I and synaptobrevin 2 were reduced in 8 -month-old $A 53 T$-transgenic mice, and the reduction was accelerated at 12 months of age. By contrast, mice in which the $\alpha$-synuclein transgene was suppressed between 9 and 12 months of age showed a reversal of the presynaptic pathology.

Does the suppression of the $\alpha$-synuclein transgene lead to an improvement in memory function? Whereas the human A53T-mutant $\alpha$-synuclein transgene-expressing mice showed a significant impairment in contextual fear conditioning, mice in which the $\alpha$-synuclein transgene was suppressed between 9 and 12 months of age showed a reduced contextual fear score that was not significantly different from the controls.

\section{Discussion \& significance}

This report is an important step forward in elucidating the role of $\alpha$-synuclein in the pathophysiology of neurodegeneration. Four aspects are in my opinion, of great interest:

- The modeling of DLB pathology in a mouse model; 
- The analysis of the regional and disease coursedependent post-translational modification of $\alpha$-synuclein pathology;

- The correlation between presynaptic $\alpha$-synuclein pathology and memory impairment;

- The reversal of presynaptic $\alpha$-synuclein pathology and mild improvement of memory function after $\alpha$-synuclein withdrawal.

CaMKII $\alpha$ has been shown to be involved in hippocampal long-term potentiation and spatial memory [8]. This serine-threonine protein kinase is expressed in the olfactory bulb, forebrain neocortical neurons, hippocampus, basal ganglia and amygdala [8]. In combination with a tetracycline transactivator system, an elegant system has been established to achieve regional and temporal control of transgene expression [8]. The expression may vary to some extent, independent of the copy numbers in the responder lines, indicating that position effects of the integration of the transgene may be responsible for topographical differences in transgene expression $[8,9]$.

The human transgenic mouse model may be somewhat artificial since the human $\alpha$-synuclein, whose expression is induced in parallel to the physiological mouse $\alpha$-synuclein, is not necessarily a functional protein in the mouse organism. On the other hand, human A53Tmutant $\alpha$-synuclein expression under the control of the CaMKII $\alpha$ promoter causes an $\alpha$-synuclein pathology that resembles that of DLB as demonstrated by Lim and coworkers [1]. A recent publication showed a similar hippocampal $\alpha$-synuclein pathology in mossy fiber terminals of the CA3 region, as demonstrated in the report reviewed here, using the same tetracycline transactivator-coupled CaMKII $\alpha$ promoter system, but driving human wildtype $\alpha$-synuclein expression [9]. In this publication, a striatonigral involvement of the transgene expression and nigral pathology was also reported.

A spread from the forebrain to the brain stem can be assumed by the results shown in both papers, and this may indicate differences in the pathophysiology of DLB and PD. Whereas in PD a spread from the brain stem to basal ganglia and cortical structures may take place, as suggested by Braak and coworkers [10], it may start in DLB in limbic areas, successively involving the striatonigral system, brain stem and neocortical regions. However, in the present paper, the striatum, basal ganglia and thalamus were spared in the instructive illustration of the pathology that resulted from transgenic overexpression of $A 53 T$-mutant $\alpha$-synuclein under the CaMKII $\alpha$ promoter.

By using a novel antibody specific for Ser129unphosphorylated $\alpha$-synuclein parallel to an antibody against Ser-129 phosphorylated $\alpha$-synuclein, Lim and coworkers were able to show that in their transgenic mouse model, the post-translational Ser-129 phosphorylation seems to be neither a prerequisite for aggregation, nor to be the step driving $\alpha$-synuclein to aggregation [1]. The spatial distribution and the relative amount of Ser-129 phosphorylated $\alpha$-synuclein increased progressively over time, and it is predominantly seen in parallel to the development of insoluble $\alpha$-synuclein aggregates. It seems that advanced stages are characterized by $\alpha$-synuclein phosphorylation. This points towards the currently favored hypothesis that $\alpha$-synuclein phosphorylation is a protective mechanism [11]. This post-translational modification may be a detoxification step, similar to the Lewy body formation. Although detection of phosphorylated $\alpha$-synuclein in human diseases is a helpful diagnostic tool in neuropathology, we have shown with the paraffin-embedded tissue blot method in DLB brain tissue samples that Ser-129 phosphorylated $\alpha$-synuclein represent only a fraction of the $\alpha$-synuclein aggregates outside the Lewy bodies [3].

Recently, in a very elegant study, Scott and coworkers have shown that mild overexpression of human wild-type $\alpha$-synuclein leads to a marked alteration of presynaptic vesicles and a reduction of the presynaptic proteins synapsin, synaptobrevin 2, amphiphysin and Piccolo [5]. Lim and coworkers have now shown that the reduction of presynaptic vesicle proteins in mossy fibers neighboring hippocampal CA3 is a time-dependent phenomenon and thus probably associated with $\alpha$-synuclein accumulation [1]. In parallel, proteins that are localized at the synaptic plasma membrane, such as syntaxin and SNAP-25, were not diminished. Hence, the synaptic terminals themselves still appear to be present.

While the synaptic pathology in the hippocampus of the human A53T-transgenic mice was paralleled by an impaired memory, as was measured by reduced contextual fear, the suppression of the $\alpha$-synuclein transgene resulted in a reverse of the synaptic pathology and only a steady state-to-mild improvement of memory function. Here, a discrepancy between the pathological changes and the clinical presentation is 
detectable, which may be most likely explained by a postsynaptic pathology. In a post-mortem tissue analysis of DLB patients, Kramer and Schulz-Schaeffer found a dramatic reduction of dendritic spines, while the presynaptic terminals were relatively preserved, although packed with $\alpha$-synuclein aggregates [4]. Thus, the tetracycline transactivator-driven A53Tmutant human $\alpha$-synuclein-transgenic mouse model using the CaMKII $\alpha$-promoter seems to mimic the pathogenesis of human DLB very well and provides important insights into the pathophysiological processes of the $\alpha$-synuclein aggregation-related disease.

\section{Conclusion \& future perspective}

The study by Lim and coworkers raises further evidence that $\alpha$-synuclein accumulation or aggregation is causally linked to a presynaptic pathology of synaptic vesicles, and that these changes go hand-in-hand with cognitive impairment [1]. A withdrawal of $\alpha$-synuclein transgene expression can reverse synaptic pathology and can stop the progression of cognitive decline. An ongoing discussion exists regarding whether the aggregation process in neurons or the deposition of aggregates is related to the neuronal loss of function. When synaptic deposits are present after withdrawal of $\alpha$-synuclein, but the nerve cell function can be restored, then the aggregation process itself may be the harmful step. When a withdrawal of $\alpha$-synuclein leads to a reduction of presynaptic aggregates, it indicates that neurons may be able to detoxify these aggregates. Both scenarios would open the way for new therapeutic strategies.

The importance of $\alpha$-synuclein in the pathophysiology of disease supports the idea of using $\alpha$-synuclein as a biomarker. Recent studies have presented evidence for this strategy [12]. It remains unclear as to whether posttranslationally modified $\alpha$-synuclein may be accessible and useful as an additional biomarker.

\section{Financial \& competing interests disclosure}

WJ Schulz-Schaeffer serves as a consultant for Bayer Schering Pharma and has received grants from the VolkswagenStiftung, Deutsche Forschungsgemeinschaft, Alberta Prion Research Institute, Canada and the EU. The author has no other relevant affiliations or financial involvement with any organization or entity with a financial interest in or financial conflict with the subject matter or materials discussed in the manuscript apart from those disclosed.

No writing assistance was utilized in the production of this manuscript.

\section{Executive summary}

\section{Background}

- Dementia with Lewy bodies and Parkinson's disease are both related to $\alpha$-synuclein aggregation, which can be found in the form of cytoplasmic Lewy bodies, neuritic aggregates (Lewy neurites) and presynaptic aggregates.

- The link between $\alpha$-synuclein aggregation and neurodegeneration remains to be elucidated.

\section{Methods \& results}

- Mice expressing human wild-type or A53T-mutant $\alpha$-synuclein under the control of a CaMKIl $\alpha$ promoter and a tetracycline-regulated transactivator were generated as a 'tet-off' system, making the control and timing of transgene expression possible by feeding the mice with doxycycline.

- A development of $\alpha$-synuclein-related synaptic pathology was observed, which increased with the time of A53T-mutant-synuclein transgene expression and reversed after transgene withdrawal.

- The synaptic pathology was paralleled by memory impairment, which was suspended or mildly reversed after transgene withdrawal.

\section{Discussion \& significance}

- The A53T-mutant $\alpha$-synuclein transgene mouse model under the control of a CaMKIl $\alpha$ promoter models the dementia with Lewy bodies pathology that may spread from limbic areas to the neocortex and the nigrostriatal system, whereas in Parkinson's disease, a spread from the brain stem to the cortex is assumed.

- The post-translational modification of $\alpha$-synuclein aggregation seems to be a late event in the aggregation process and may be part of the neuronal defense against aggregate toxicity.

- To explain the discrepancy between reversal of synaptic pathology and restoration of memory, postsynaptic pathological changes should be considered.

\section{Conclusion \& future perspective}

- The study provides evidence for a link between $\alpha$-synuclein accumulation or aggregation and presynaptic vesicle pathology.

- Elucidating the pathomechanism of $\alpha$-synuclein-related synaptic pathology may open up the possibility of new therapeutic approaches.

- The study supports the idea of using $\alpha$-synuclein as a biomarker. 


\section{References}

1. Lim Y, Kehm VM, Lee EB et al. Alpha-Syn suppression reverses synaptic and memory defects in a mouse model of dementia with Lewy bodies. J. Neurosci. 31(27), 10076-10087 (2011).

2. Spillantini MG, Schmidt ML, Lee VM, Trojanowski JQ, Jakes R, Goedert M. Alpha-synuclein in Lewy bodies. Nature 388(6645), 839-840 (1997).

3. Schulz-Schaeffer WJ. The synaptic pathology of $\alpha$-synuclein aggregation in dementia with Lewy bodies, Parkinson's disease and Parkinson's disease dementia. Acta Neuropathol. 120(2), 131-143 (2010).

4. Kramer ML, Schulz-Schaeffer WJ. Presynaptic alpha-synuclein aggregates, not Lewy bodies, cause neurodegeneration in dementia with Lewy bodies. J. Neurosci. 27(6), 1405-1410 (2007).
5. Scott DA, Tabarean I, Tang Y, Cartier A, Masliah E, Roy S. A pathologic cascade leading to synaptic dysfunction in alphasynuclein-induced neurodegeneration. J. Neurosci. 30(24), 8083-8095 (2010).

6. Nemani VM, Lu W, Berge V et al. Increased expression of alpha-synuclein reduces neurotransmitter release by inhibiting synaptic vesicle reclustering after endocytosis. Neuron 65(1), 66-79 (2010).

7. Lim Y, Kehm VM, Li C, Trojanowski JQ, Lee M. Forebrain overexpression of alphasynuclein leads to early postnatal hippocampal neuron loss and synaptic disruption. Exp. Neurol. 221(1), 86-97 (2010).

8. Mayford M, Bach ME, Huang YY, Wang L, Hawkins RD, Kandel ER. Control of memory formation through regulated expression of a CaMKII transgene. Science 274(5293), 1678-1683 (1996).
9. Nuber S, Petrasch-Parwez E, Winner B et al. Neurodegeneration and motor dysfunction in a conditional model of Parkinson's disease. J. Neurosci. 28(10), 2471-2484 (2008).

10. Braak H, Del TK, Rub U, de Vos RA, Jansen Steur EN, Braak E. Staging of brain pathology related to sporadic Parkinson's disease. Neurobiol. Aging 24(2), 197-211 (2003).

11. Oueslati A, Fournier M, Lashuel HA. Role of post-translational modifications in modulating the structure, function and toxicity of alpha-synuclein: implications for Parkinson's disease pathogenesis and therapies. Prog. Brain Res. 183, 115-145 (2010).

12. Mollenhauer B, Locascio JJ, Schulz-Schaeffer W, Sixel-Doring F, Trenkwalder C, Schlossmacher MG. alpha-synuclein and tau concentrations in cerebrospinal fluid of patients presenting with parkinsonism: a cohort study. Lancet Neurol. 10 (3), 230-240 (2011). 